ablative therapy. Thus, the positive predictive value of cervical cytology [Pap screening] for underlying HSIL has reduced: accordingly, countries are adopting new screening strategies using more sensitive, more objective methods of HPV nucleic acid tests (NAT). In 2017 Australia, screening changed to HPV NAT assays commencing at 25 years of age, with immediate triage to cytology and colposcopy if HPV16/18 positive, and five yearly screening for those HPV DNA negative. It is important that NAT assays chosen must strike a balance between sufficient clinical sensitivity to detect/predict HSIL, without being too sensitive (detecting transient infection only not destined to becoming lesions). The highest quality HPV NAT is thus a priority to reduce falsely negative screens and manage the risk associated with false positive HPV NAT test results. It is imperative that we adopt the best QA and QC measures to accompany the introduction of these new assays. Today we are poised to markedly reduce the incidence of cervical cancer, with the vision of eventually eliminating it as a public health problem (as the call to action from WHO DG Dr Tedros May 2018), using the combination of sustained high coverage HPV vaccination and sustained high coverage HPV NAT screening, with treatment of those with disease.

Disclosure No significant relationships.

\section{S17.2 TREATMENT OF BACTERIAL VAGINOSIS: HOW, WHEN AND HOW MUCH?}

Caroline Mitchell ${ }^{*}$. Massachusetts General Hospital, Obstetrics and Gynecology, Boston, USA

\subsection{6/sextrans-2019-sti.78}

Bacterial vaginosis (BV) is a leading cause of symptomatic vaginitis in North American populations. BV is also associated with adverse reproductive health outcomes such as miscarriage, preterm birth, and increased risk for sexually transmitted diseases. Treatment of this common disorder is mostly effective in the short term, but recurrence is common. Current treatment guidelines will be reviewed, as well as alternative regimens and treatments, some of which may not be FDAapproved for treating BV. This presentation will discuss the short and long term efficacy of recommended antibiotic treatment regimens, as well as alternative treatment and prevention strategies such as boric acid and probiotics. The role of treatment for prevention of adverse health outcomes will be reviewed. Predictors of treatment success will be discussed, and incorporated into practical advice for patients and treating providers.

Disclosure No significant relationships.

\section{S17.3 SCREENING WOMEN FOR BACTERIAL STIS: SHOULD WE SCALE-BACK?}

Jonathan Ross*. University Hospital Birmingham NHS Trust, Birmingham, UK

10.1136/sextrans-2019-sti.79

Screening for bacterial STIs, in particular chlamydia, is common in high income countries using a variety of different approaches. However, recent reductions in funding for STI treatment services in several countries highlight the need to ensure that investment in screening remains appropriate and cost-effective. The evidence base for chlamydia screening suggests that it can reduce the incidence of pelvic inflammatory disease but with several caveats, and it remains unclear whether screening represents good value for money. In particular, the opportunity cost of choosing screening over other sexual health interventions requires consideration. Harms have been poorly characterised but need to be addressed when measuring the potential value of a screening program. Overall, the evidence for broad population based screening is limited and new approaches are needed to reduce the morbidity and reproductive sequelae associated with bacterial STIs.

Disclosure No significant relationships.

\section{S17.4 PREMATURITY AND STI - VALUE OF SCREENING AND TREATMENT}

Deborah Money*. University of British Columbia, Vancouver, Canada

\subsection{6/sextrans-2019-sti.80}

Prematurity and STI - the value of screening and treatment Preterm birth is a significant cause of perinatal morbidity and mortality worldwide and strategies to prevent preterm birth have often focussed on infectious etiologies. In addition, prenatal care is a time when women access health care, even in resource limited settings, and poses a very important opportunity to optimize a woman's health and ensure screening for communicable diseases, particularly STI's is conducted. Screening and treating STI's in any reproductive aged woman are important but treating in pregnancy requires appropriate selection of antimicrobials that will be the safest in pregnancy depending on the gestational age. Many STI's have been associated with preterm birth but treating in all cases has not been consistently associated with prevention of prematurity. This talk will present data on the most common STI's and sexually associated genital tract infections, and the individual value of screening and treating in pregnancy including; HIV, Hepatitis B, Hepatitis C, Syphilis, Herpes simplex, Trichomonas vaginalis, bacterial vaginosis, Chlamydia trachomatis, Neisseria gonorrheae, Mycoplasma genitalium, Mycoplasma hominus, Ureaplasma urealyticum. Screening and treatment of bacterial vaginosis has been studied extensively over time with variability in results. Many studies have shown an association of bacterial vaginosis with higher rates of preterm birth, but in low risk women treatment has not proven of benefit. In some studies, treatment of high-risk women has been beneficial. However, the lack of precision around the diagnosis of bacterial vaginosis has likely contributed to confusing results in the literature, and new methods of genomic analysis of the vaginal microbiome is leading to opportunities for much greater precision in the diagnosis of dysbiosis and targeted treatment trials that would be more promising than standard treatment has proven to be in the past.

Disclosure No significant relationships. 